\title{
Evolution mechanism of crystallographic orientation in grain continuator bars of a Ni-based single-crystal superalloy prepared by Bridgman technology during directional solidification
}

\author{
Jiu-han Xiao", ${ }^{1,}$ *Wei-guo Jiang ${ }^{1,3}$, Dong-yu Han ${ }^{1,2}$, Kai-wen Li', Guo-jun Tong ${ }^{\mathbf{4}}$, Yu-zhang Lu', and Lang-hong Lou' \\ 1. Shi-Changxu Innovation Center for Advanced Materials, Institute of Metal Research, Chinese Academy of Sciences, Shenyang 110016, China \\ 2. School of Materials Science and Engineering, University of Science and Technology of China, Shenyang 110016, China \\ 3. Weifang University of Science and Technology, Shouguang 262700, Shandong, China \\ 4. Powerplant Engineering Technical Managements Division, Shenyang Maintenance \& Overhaul Base China Southern Airlines, Shenyang \\ 110000, China
}

\begin{abstract}
Single-crystal rods with different diameters and deviation angles with respect to the solidification direction were produced by Bridgman rapid solidification method at withdrawal rates of 3 and $6 \mathrm{~mm} \cdot \mathrm{min}^{-1}$ and used as grain continuators. The crystallographic orientation of the rods, which cross-sections were perpendicular to the solidification direction at different solidification heights, was measured by electron backscattered diffraction, while the corresponding microstructures were observed by optical microscopy. The mushy zone morphology and the distribution of the temperature gradient were simulated by the finite element analysis software ProCAST. The experimental results indicate that the crystallographic orientation of the single-crystal rods corresponds to the statistical average value of all the dendrite orientations in cross-section. The crystallographic orientation of the primary and secondary dendrites of each single-crystal rod at different cross-sections fluctuates irregularly within a small range (less than $4^{\circ}$ ). The crystallographic orientation of the dendrite in each single-crystal rod is not exactly consistent with each other and is affected by their branching mode of dendrites in the solidification space. In addition, the simulation results show that the mushy zone shapes and the temperature gradient of single-crystal rods change with the increase of solidification height during the solidification process. Finally, the evolution mechanism of the crystallographic orientations and the corresponding influence factors were analyzed and discussed.
\end{abstract}

Keywords: single-crystal superalloy; dendrite growth; orientation evolution; solidification space; withdrawal rate; cross-section size
CLC numbers: TG132.3 3
Document code: A
Article ID: 1672-6421(2022)01-035-11

\section{Introduction}

Ni-based single-crystal turbine blades have been used extensively in advanced aero-engines and landbased industrial gas turbines due to their excellent mechanical properties and microstructure stability at elevated temperatures, profiting from the elimination of transverse grain boundary defects ${ }^{[1]}$. Generally, single-crystal turbine blades are prepared by directional

\footnotetext{
*Wei-guo Jiang
}

Born in 1968, Ph. D, Professor. His research interests mainly focus on the nickel-based superalloy design, solidification defects, numerical simulation, and ceramic core technology development.

E-mail: jwg@wfust.edu.cn

Received: 2021-07-28; Accepted: 2021-12-14 solidification combined with selecting grains or "seeding" technology ${ }^{[2-4]}$. However, solidification defects, mainly including low-angle boundaries (LABs), freckles, slivers, and stray grains ${ }^{[5-9]}$, are usually observed due to the local instability of the temperature field and solute field in the blade platforms. These defects result in an obvious deterioration of the blades' performance. To avoid the solidification defects mentioned above, a grain continuator (GC) technique was developed to prepare the single-crystal turbine blades ${ }^{[10-12]}$.

GCs can guide the primary crystal of the blades to grow into the target position in the platform (defined as "GC crystal"), followed by converging with the primary crystal grown from the blades. The GC technique can also serve as a special seeding technology. The specialty of the GC technique is that 
the seed crystal is formed during the directional solidification and not pre-placed in the base of the turbine blade's ceramic shell mold. Normally, the installation direction of the GCs is not strictly in line with the single-crystal solidification direction due to the complex geometry of the blades. Nonetheless, the deviation angles between the GCs and the solidification direction should be limited at a suitable range to obtain perfect single-crystal turbine blades ${ }^{[13]}$. In theory, the orientation of the GCs is similar to that of the blades along directional solidification. However, under better cooling conditions, the progression of the solidification front in the GCs is faster than in the blades. This may cause the dendrites to deviate or twist inside the GCs, different from those in the blades during directional solidification ${ }^{[10,14]}$. As a result, the converging interface between the GC crystal and the primary crystal in the platform may present a certain misorientation. To sum up, the misorientation of the converging interface is determined by the orientation consistency of the GC crystal and the primary crystal during directional solidification. When this misorientation gets to a higher value (e.g., exceeds $6^{\circ}$ ), it will seriously damage the properties of the blades ${ }^{[15-17]}$.

Some researchers have investigated the evolution mechanism of the crystallographic orientation of single-crystal superalloys during directional solidification. Newell et al. ${ }^{[18]}$ reported that during the growth of single-crystal, there is no rotation pattern from the [001] axis along the solidification direction: the rotation is random. In contrast, the experimental results of $\mathrm{Hu}$ et al. ${ }^{[19]}$ indicate that the orientation of single-crystal casting deviated along the solidification direction. To date, few studies have focused on the influence of the solidification space deviation angle from the solidification direction, the withdrawal rate, and the cross-section dimensions of castings on the orientation evolution in GCs. Hence, the evolution mechanism of the crystallographic orientation of the GCs needs to be deeply understood.

In this work, single-crystal GC rods with different diameters and deviation angles from the solidification direction were designed to determine the characteristics of the crystallographic orientation evolution. The directional solidification process of the single-crystal rods was simulated by ProCAST software. The crystallographic orientations of the GC rods at different growth heights, as well as their microstructures, were obtained to confirm the development process of crystallographic orientation, and to discuss the evolution mechanism of the crystallographic orientation in space.

\section{Experimental procedure}

To investigate the evolution of the crystallographic orientation in different GCs during directional solidification, two groups of GC rods were designed [Fig. 1(a)]: vertical rod (VR) samples and tilted rod (TR) samples, and the deviation angles from solidification direction [001] were $0^{\circ}$ and $15^{\circ}$, respectively. Both group samples included four different cross-section sizes, with diameters of $3 \mathrm{~mm}, 5 \mathrm{~mm}, 8 \mathrm{~mm}$, and $10 \mathrm{~mm}$, respectively. A wax pattern cluster is shown in Fig. 1(b). The surfaces of the samples facing the central sprue and the graphite heater were defined as "inside" and "outside", respectively. An investment casting process was performed to produce a ceramic shell mold. The wax pattern cluster was dipped into $\mathrm{Al}_{2} \mathrm{O}_{3}$ slurries and stuccoed by $\mathrm{Al}_{2} \mathrm{O}_{3}$ sands with different particle sizes. This procedure was repeated to ensure that the thickness of the ceramic shell wall is $6 \mathrm{~mm}$. Dewaxing was carried out in a steam autoclave at $170{ }^{\circ} \mathrm{C}$ under $6 \mathrm{~atm}$ pressure for $15 \mathrm{~min}$. Then, the shell mold was air-baked at $900{ }^{\circ} \mathrm{C}$ for $4 \mathrm{~h}$ to burn out the residual wax and enhance its strength. The Bridgman rapid solidification technique was applied for preparing singlecrystal superalloy rods. A Ni-based single-crystal superalloy was employed in the present experiment. Its chemical composition (wt.\%) was Ni-12.5Cr-9Co-3.7W-1.9Mo-2.8Al$3.4 \mathrm{Ti}-5 \mathrm{Ta}$ and $0.08 \mathrm{C}$. The temperatures of the pouring molten superalloy and the heater were set at $1,520^{\circ} \mathrm{C}$ and $1,500{ }^{\circ} \mathrm{C}$, respectively. Two different constant withdrawal rates $(3$ and $6 \mathrm{~mm} \cdot \mathrm{min}^{-1}$ ) were used in the directional solidification processes. The spiral grain selection method was performed to obtain the single-crystal GC rods in approximately the [001] direction. (a)

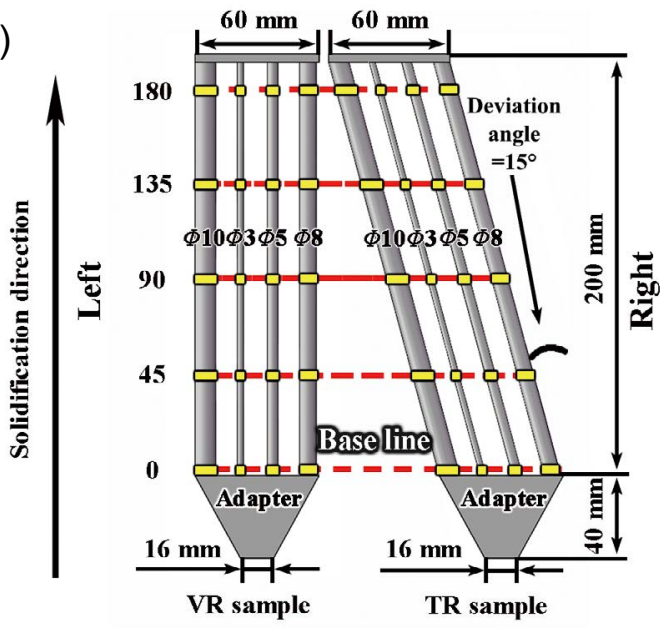

(b)

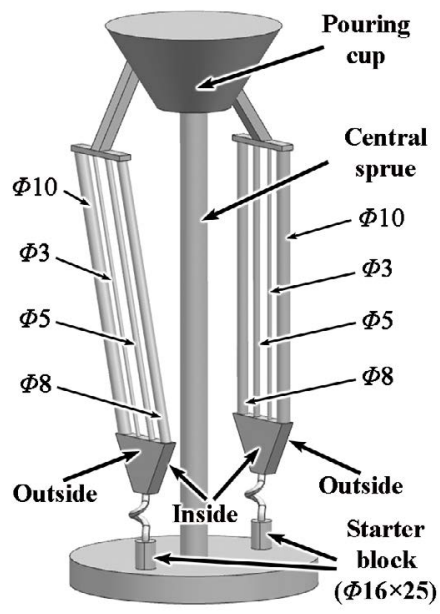

Fig. 1: Geometry of grain continuators (a) and schematic diagram of wax pattern cluster (b) 
After directional solidification, the ceramic shell was removed, and the castings were obtained after cutting runners and the sand-blasting treatment. Samples with a thickness of $2 \mathrm{~mm}$ were machined at solidification heights of $0 \mathrm{~mm}, 45 \mathrm{~mm}, 90 \mathrm{~mm}$, $135 \mathrm{~mm}$, and $180 \mathrm{~mm}$ from the top of the adapter [indicated by yellow dots in Fig. 1(a)] by wire electrical discharge machining. It should be noted that two parallel samples were obtained for measuring primary orientation and secondary orientation at the same solidification height, respectively. Then, the samples were ground, polished, and vibratory polished for surface stressrelief. The treated surfaces for measuring primary orientation were perpendicular to the solidification direction, and those for secondary orientation were parallel to the solidification direction. Finally, the crystallographic orientation was determined by the electron backscattered diffraction (EBSD) method. All the surfaces of the samples from the same rod were placed parallel to each other. The EBSD measurements were carried out by using a ZEISS Merlin Compact field emission-scanning electron microscope equipped with a NordlysNano detector (Oxford Instruments). Notably, a step size of $80 \mu \mathrm{m}$ was applied for the data acquisition. To ensure the accuracy of the EBSD measurements, a single-crystal silicon was used to characterize the orientation precision. Moreover, the "refined accuracy" mode of the Aztec software (Oxford Instruments) was adopted to improve the angular resolution. Subsequently, Channel 5 software was applied for analyzing the EBSD data. Microstructure observations were performed by using an optical microscope (Zeiss Axio Vert. A1). The metallographic microstructure and the crystallographic orientation of the longitudinal section (which

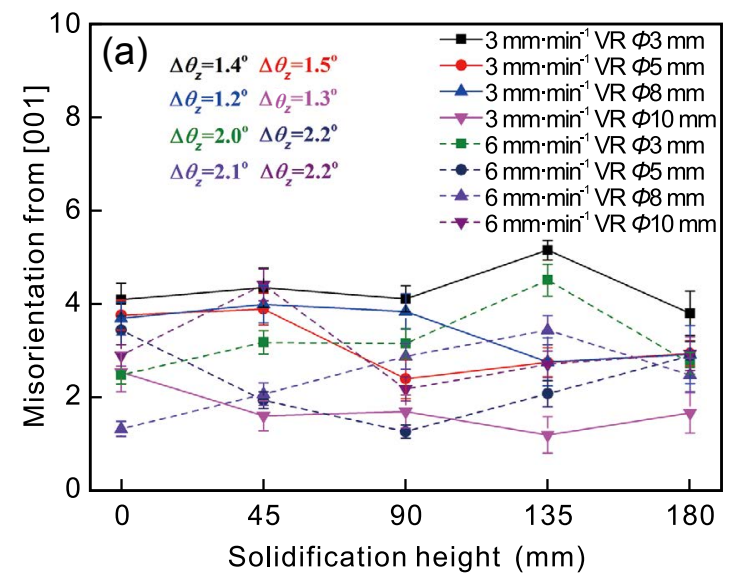

was parallel to the solidification direction) of the samples were analyzed at solidification heights of $0 \mathrm{~mm}, 90 \mathrm{~mm}$ and $180 \mathrm{~mm}$. Meanwhile, the commercial finite element software ProCAST was utilized to simulate the directional solidification processes of the samples to assist the analysis of the crystallographic orientation evolution. The specific parameters of the boundary conditions and initial conditions used for the numerical simulations were the same as Ref. [5].

\section{Results}

The crystallographic orientations of the primary and secondary dendrites were defined as "primary" and "secondary" orientations, respectively ${ }^{[20]}$. The primary crystallographic orientations of the VR and TR samples at different heights and withdrawal rates are shown in Fig. 2. Owing to the adoption of the spiral grain selection method, the samples with primary orientation close to [001] direction were obtained. At different withdrawal rates, the primary orientations in the VR and TR samples deviated gradually from the [001] direction with the increase of the solidification height. Here, the orientation deviation range is indicated by " $\Delta \theta_{\mathrm{Z}}$ ". $\Delta \theta_{\mathrm{Z}}$ is equal to the difference between the maximum misorientation and the minimum misorientation among all sections at different heights. The maximum $\Delta \theta_{\mathrm{Z}}$ value of the experimental samples is $3.2^{\circ}$, as shown in Fig. 2(b). Meanwhile, it can be observed that the crystallographic orientations are not significantly affected by the cross-section sizes. The deviation range of the orientation increases slightly with the increase of the withdrawal rate.

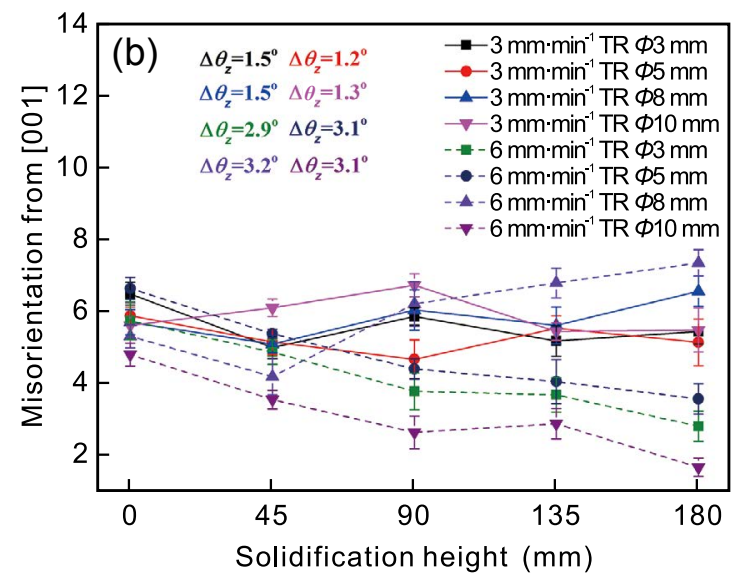

Fig. 2: Evolution of primary dendrite orientation along the solidification direction at different withdrawal rates: (a) VR sample; (b) TR sample

The crystallographic orientation in different regions of the same section (at a solidification height of $45 \mathrm{~mm}$ ) of the TR rod with a $3 \mathrm{~mm}$ diameter and at a withdrawal rate of $6 \mathrm{~mm} \cdot \mathrm{min}^{-1}$ was examined. The orientation map and corresponding inverse pole figure (IPF) are shown in Figs. 3(a) and (b1), respectively. Four subset regions and the corresponding IPFs are shown in Figs. 3(b2-b5). The position and distribution of the orientation points in the IPF are not entirely consistent with each other in different zones. The orientation of the whole cross-section corresponds to the sum of the orientations of the four subset regions. Similar results have been previously reported ${ }^{[21]}$.

Similarly to the evolution results of the primary orientation along the [001] direction, the orientation of the secondary dendrite is also deviated, as shown in Fig. 4. However, the initial orientation of the secondary dendrite is random in space, due to an inability to control the secondary orientation by spiral grain selection method. $\Delta \theta_{X}$ represents the deviation range of secondary orientation. Here, $\Delta \theta_{X}$ equals to the difference between the maximum misorientation and the minimum misorientation of the secondary dendrite among sections perpendicular to the 
(a)

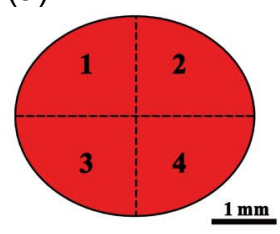

(b3)

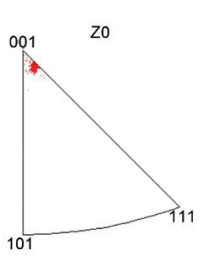

(b1)

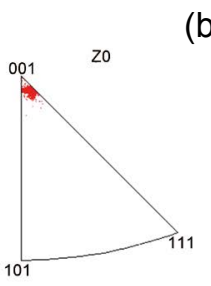

(b4)

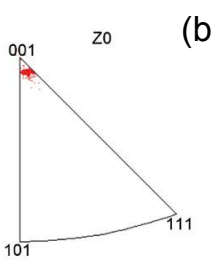

(b2)

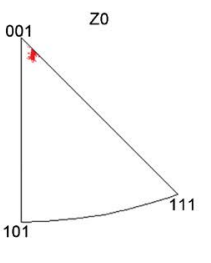

(b5)

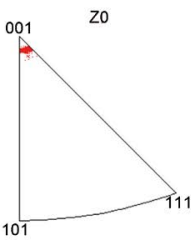

Fig. 3: Orientation map and inverse pole figure (IPF) of $\Phi 3 \mathrm{~mm}$ TR sample at solidification height of $45 \mathrm{~mm}$ with withdrawal rate of $6 \mathrm{~mm} \cdot \mathrm{min}^{-1}$ : (a) orientation map; (b1) IPF of whole cross-section; (b2) IPF of Region 1; (b3) IPF of Region 2; (b4) IPF of Region 3; (b5) IPF of Region 4

(a)

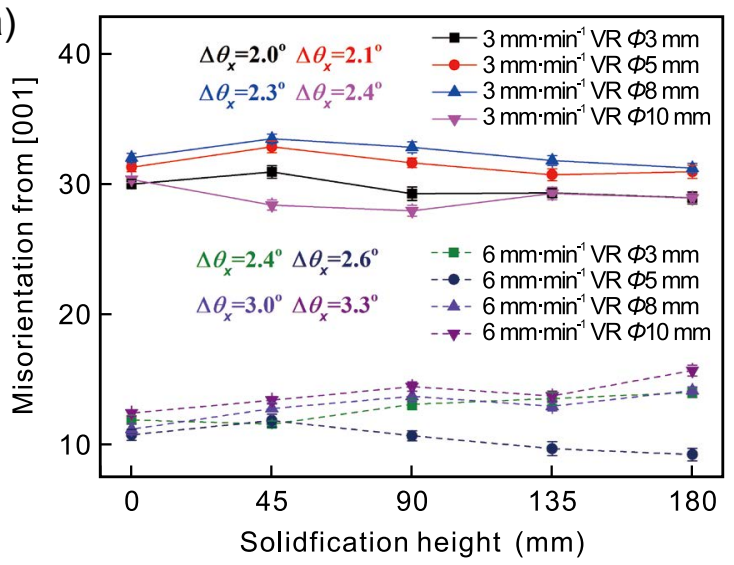

solidification direction at different heights. The maximum value of $\Delta \theta_{X}$ is $3.6^{\circ}$ : fairly close to that of $\Delta \theta_{Z}\left(3.2^{\circ}\right)$. With the increase of the section size and withdrawal rate, the $\Delta \theta_{X}$ value increases slightly.

From the above crystallographic orientation evolution results, it is evident that fluctuant orientation evolution phenomena were verified under all the tested experimental conditions.

The typical evolution results of the microstructure morphology and the corresponding primary dendrite orientation at heights of $0 \mathrm{~mm}, 90 \mathrm{~mm}$, and $180 \mathrm{~mm}$ under a withdrawal rate of $6 \mathrm{~mm} \cdot \mathrm{min}^{-1}$ are shown in Fig. 5. The orientations of the central dendrites (solid red arrows) are significantly different from those of the dendrites on the left edge (solid yellow arrows) and right edge (solid white arrows) in both the VR [Fig. 5(a)] and TR [Fig. 5(b)] samples. The misorientations between the dendrites in the center (as the base) and on the left or right edge are indicated with $\theta_{\mathrm{LM}}$ and $\theta_{\mathrm{RM}}$, respectively. The values of $\theta_{\mathrm{LM}}$ and $\theta_{\mathrm{RM}}$ are inconsistent in different samples at the

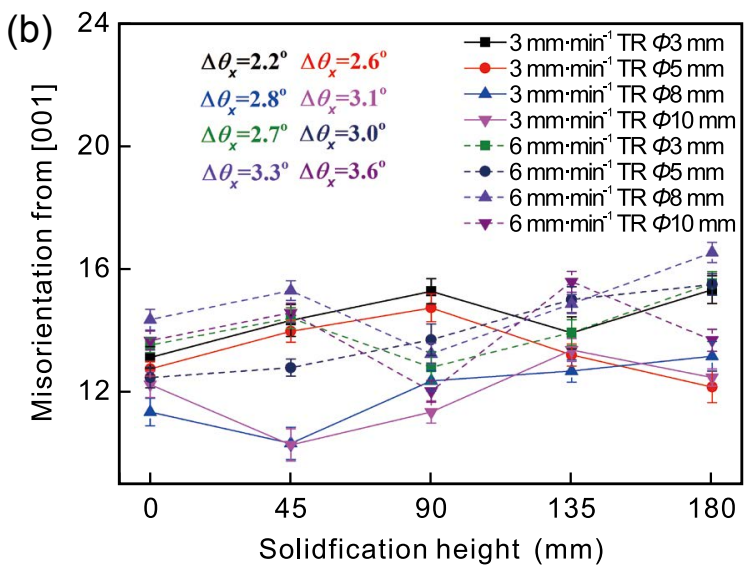

Fig. 4: Evolution results of secondary dendrite orientation at different withdrawal rates: (a) VR sample; (b) TR sample

same height (Table 1). In addition, the orientation of all dendrites changes in a specific direction monotonously and continuously. The typical propagation and elimination of dendrites (in the green boxes) were found by observing the microstructure of the sample with a diameter of $10 \mathrm{~mm}$ at heights of $0 \mathrm{~mm}$ and $90 \mathrm{~mm}$. The primary dendrite, secondary dendrite, and tertiary dendrite are abbreviated as $\mathrm{PD}, \mathrm{SD}$, and $\mathrm{TD}$, respectively. A competitive growth of dendrites (i.e., $\mathrm{PD}_{1}$ branching out into $\mathrm{SD}_{1}$ and $\mathrm{TD}_{1}$, and $\mathrm{SD}_{2}$ being blocked by $\mathrm{PD}_{1}$ and $\mathrm{TD}_{1}$ ) was observed in the enlarged views of Regions A1 and A2 of $\Phi 10 \mathrm{~mm}$ VR sample. As well, the propagation (i.e., $\mathrm{PD}_{1}$ branching out into $\mathrm{SD}_{1}$ and $\mathrm{TD}_{1}$ ) and the elimination (i.e., $\mathrm{PD}_{1}$ being blocked by the mold shell) of dendrites could also be clearly observed in the enlarged views of Regions B1 and B2 of the $\Phi 10 \mathrm{~mm}$ TR sample.

Figures 5(c) and (d) depict the IPFs of the crystallographic orientations in the VR and TR samples at different heights. The yellow circle highlights a view of the region near the [001] orientation points in the IPF. Numerous orientation points are close to the [001] orientation. Moreover, the larger the diameter of the cross-section, the more scattered the distribution of the orientation points. By comparing the position of the orientation points in the IPF at different heights, it is noticed that the spatial distribution and the range of the orientation points change slightly; moreover, a small region with overlapping orientation points could also be observed. This indicates that the primary crystallographic orientation fluctuates within a limited range. These results are in agreement with the evolution results regarding the orientation along the solidification direction (Fig. 2).

The orientation evolution of a single primary dendrite in rods with different solidification deviation angles and at a withdrawal rate $6 \mathrm{~mm} \cdot \mathrm{min}^{-1}$ was determined (Fig. 6). The single-dendrites marked by the yellow dashed arrow were tested [Figs. 6(a) and (c)]. It can be seen that the misorientation (based on the initial orientation) fluctuates as the solidification height increases. The fluctuation ranges of the primary orientation in the VR and TR samples are $0.75^{\circ}$ and $0.68^{\circ}$, respectively [Figs. 6(b) and (d)]. These results indicate that the orientation evolution of the single-dendrites along the solidification direction is unstable. 
(a)

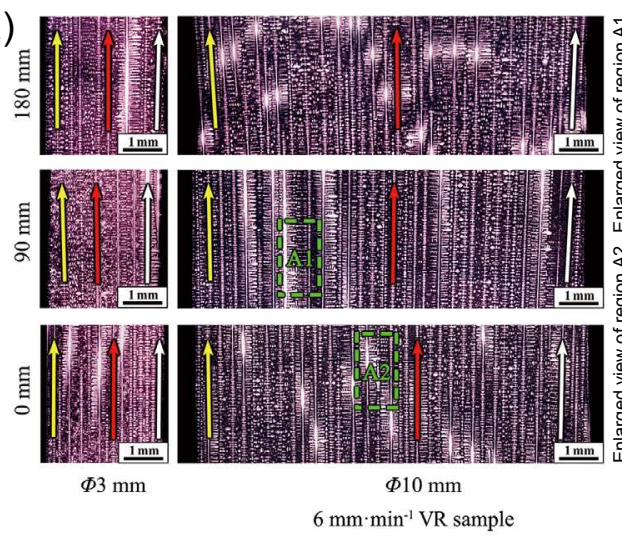

(c)

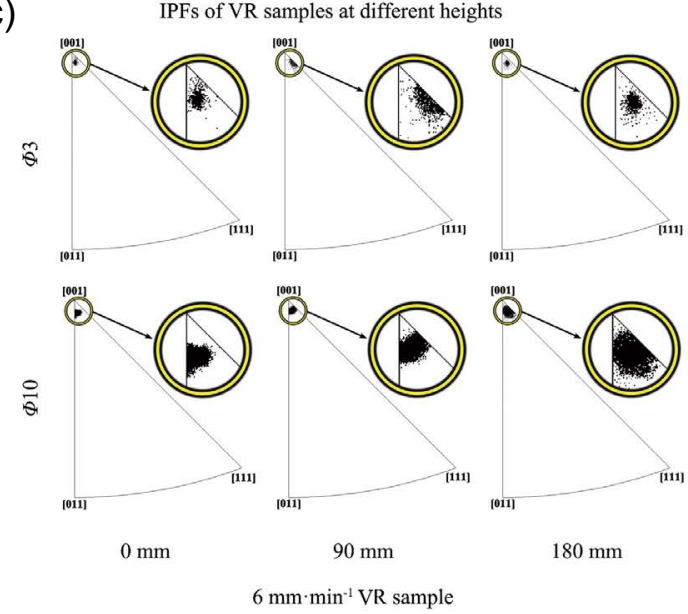

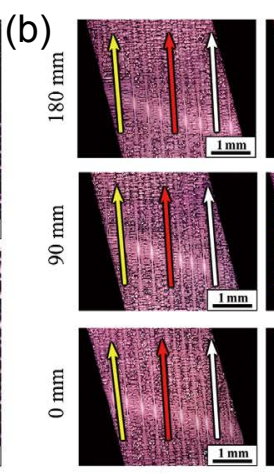
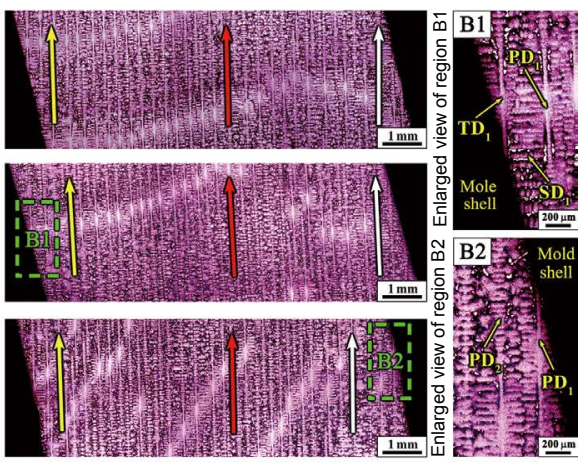

$\Phi 3 \mathrm{~mm}$

$\Phi 10 \mathrm{~mm}$

$6 \mathrm{~mm} \cdot \mathrm{min}^{-1}$ TR sample

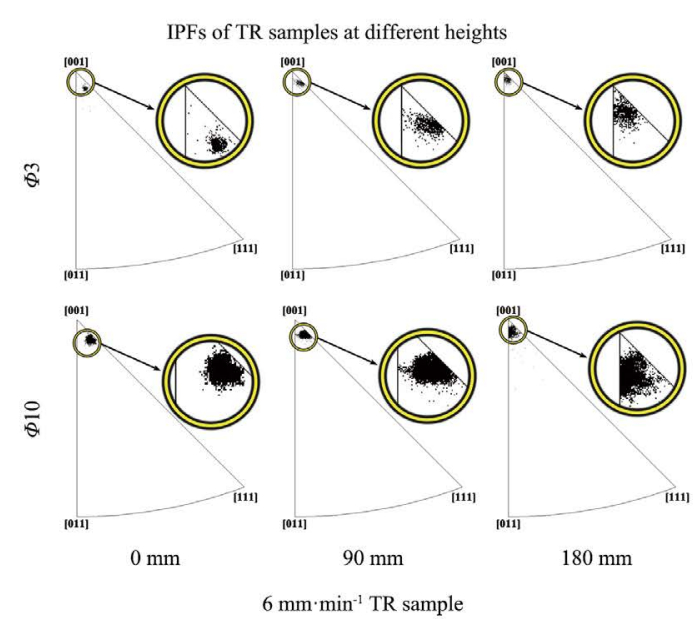

Fig. 5: Evolution results of microstructure and corresponding inverse pole figure of primary orientation at withdrawal rate of $6 \mathrm{~mm} \cdot \mathrm{min}^{-1}$ for VR $(a, c)$ and TR sample (b, d)

Table 1: Misorientation between dendrites in center (as the base) and dendrites on edges at $6 \mathrm{~mm} \cdot \mathrm{min}^{-1}$ withdrawal rate

\begin{tabular}{ccccccccc} 
Solidification & \multicolumn{2}{c}{ VR $\Phi 3$} & \multicolumn{2}{c}{ VR $\phi 10$} & \multicolumn{2}{c}{ TR $\Phi 3$} & \multicolumn{1}{c}{ TR $\phi 10$} \\
\hline height $(\mathrm{mm})$ & $\boldsymbol{\theta}_{\mathrm{LM}}$ & $\boldsymbol{\theta}_{\mathrm{RM}}$ & $\boldsymbol{\theta}_{\mathrm{LM}}$ & $\boldsymbol{\theta}_{\mathrm{RM}}$ & $\boldsymbol{\theta}_{\mathrm{LM}}$ & $\boldsymbol{\theta}_{\mathrm{RM}}$ & $\boldsymbol{\theta}_{\mathrm{LM}}$ & $\boldsymbol{\theta}_{\mathrm{RM}}$ \\
\hline 0 & 0.1 & 0.2 & 0.5 & 1.3 & 0.6 & 0.1 & 0.7 & 0.5 \\
90 & 0.5 & 1.1 & 1.7 & 2.2 & 0.2 & 0.2 & 0.2 & 0.5 \\
180 & 0.5 & 0.7 & 3.6 & 2.7 & 0.2 & 0.6 & 0.2 & 0.1
\end{tabular}

Figure 7 shows the ProCAST simulation results for the VR and TR samples considering different solidification heights and withdrawal rates. It is found that the shape and thickness of the mushy zone keep changing during directional solidification. The shape of the mushy zone is more tilted and concave in the TR sample than in the VR sample at the same withdrawal rate. The liquidus isotherms of the inside indicate higher values than those of the outside for the VR sample, while the liquidus isotherms of the left side indicate lower values than those of the right side for the TR sample. In addition, the isotherms of $\Phi 10 \mathrm{~mm}$ VR sample are more tilted and concave than those of the $\Phi 3 \mathrm{~mm}$ VR sample at the same withdrawal rate. More tilted and concave liquidus isotherms could be observed with the increase of the withdrawal rate. Here, the thickness of the mushy zone at solidification heights of $0 \mathrm{~mm}, 90 \mathrm{~mm}$, and
$180 \mathrm{~mm}$ was expressed as $L_{0}, L_{90}$, and $L_{180}$, respectively. The thicknesses of mushy zone of different samples are listed in Table 2. The mushy zone thickness is the average value of five measurements.

According to the results simulated by ProCAST, the thickness of the mushy zone in the VR sample decreases at first and then increases during directional solidification at the same withdrawal rate. However, the thickness of the mushy zone decreases with the increase of the solidification height in the TR sample. Meanwhile, for the same deviation angle and withdrawal rate, the mushy zone thickness in the $\Phi 10 \mathrm{~mm}$ sample is greater than that in the $\Phi 3 \mathrm{~mm}$ sample at the same solidification height. In the same sample, at the same solidification height, the mushy zone thickness at a withdrawal rate of $6 \mathrm{~mm} \cdot \mathrm{min}^{-1}$ is greater than that at a withdrawal rate of $3 \mathrm{~mm} \cdot \mathrm{min}^{-1}$. 


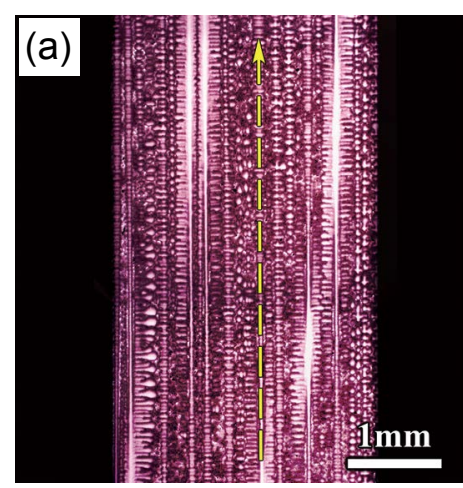

(b)

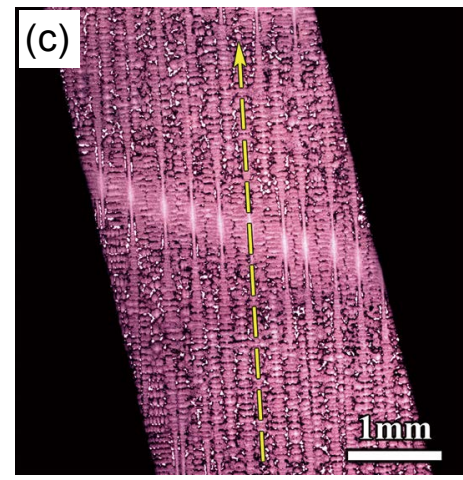

(d)
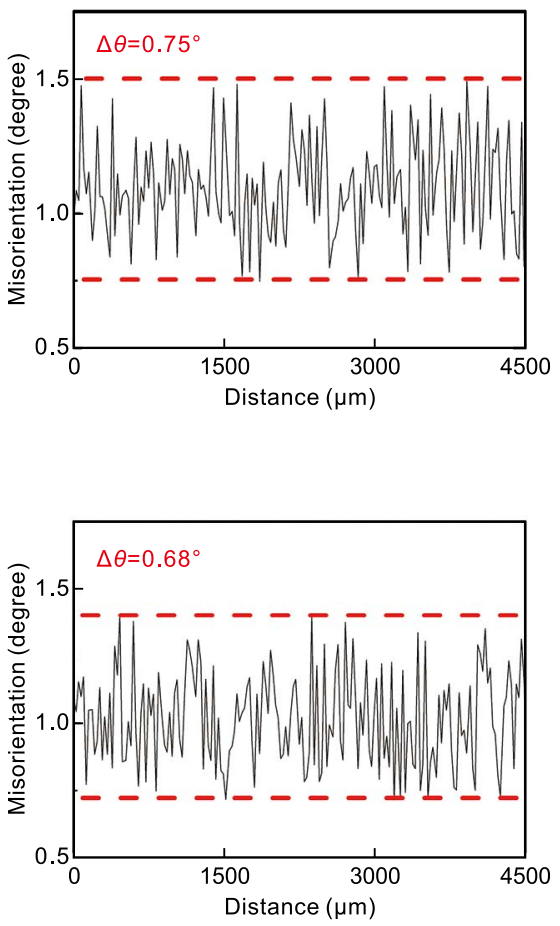

Fig. 6: Evolution results of single-dendrite at the initial height of $45 \mathrm{~mm}$ along solidification direction in $\Phi 3 \mathrm{~mm}$ samples under withdrawal rate of $6 \mathrm{~mm} \cdot \mathrm{min}^{-1}$ : (a) microstructure morphology of VR sample; (b) corresponding primary orientation evolution of VR sample; (c) microstructure morphology of TR sample; (d) corresponding primary orientation evolution of TR sample
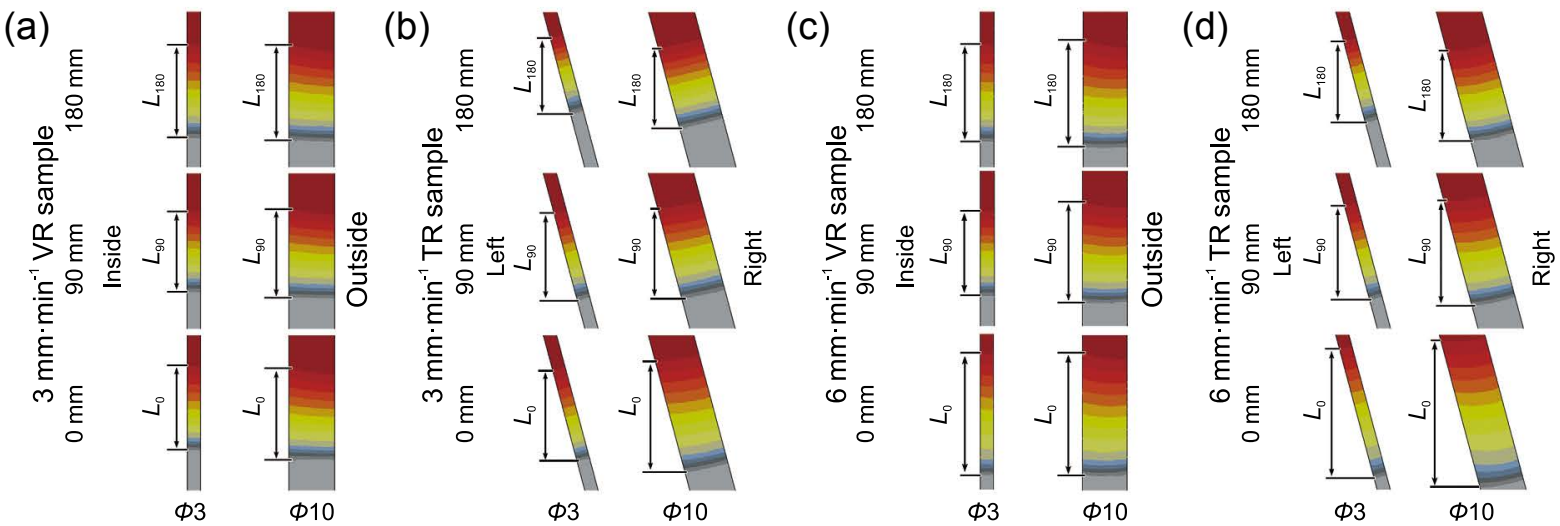

Fig. 7: Mushy zone shapes and thicknesses of VR sample, $3 \mathrm{~mm} \cdot \mathrm{min}^{-1}(\mathrm{a})$, TR sample, $3 \mathrm{~mm} \cdot \mathrm{min}^{-1}(\mathrm{~b})$, VR sample, $6 \mathrm{~mm} \cdot \mathrm{min}^{-1}(\mathrm{c})$, and TR sample, $6 \mathrm{~mm} \cdot \mathrm{min}^{-1}(\mathrm{~d})$

Table 2: Mushy zone thicknesses of samples at different withdrawal rates calculated and tested by ProCAST software

\begin{tabular}{|c|c|c|c|c|c|}
\hline \multirow{2}{*}{$\begin{array}{l}\text { Withdrawal } \\
\text { rate }\left(\mathrm{mm} \cdot \mathrm{min}^{-1}\right)\end{array}$} & \multirow{2}{*}{$\begin{array}{l}\text { Solidification } \\
\text { height (mm) }\end{array}$} & \multicolumn{4}{|c|}{ Thickness of mushy zone (mm) } \\
\hline & & VR $\Phi 3$ & VR $\Phi 10$ & TR $\Phi 3$ & TR $\Phi 10$ \\
\hline \multirow{4}{*}{3} & 0 & $18.8 \pm 0.3$ & $20.0 \pm 0.4$ & $19.5 \pm 0.3$ & $24.3 \pm 0.6$ \\
\hline & 90 & $17.7 \pm 0.2$ & $18.2 \pm 0.3$ & $19.0 \pm 0.3$ & $19.7 \pm 0.4$ \\
\hline & 180 & $20.3 \pm 0.4$ & $20.8 \pm 0.4$ & $16.4 \pm 0.2$ & $17.2 \pm 0.2$ \\
\hline & 0 & $26.5 \pm 0.7$ & $26.9 \pm 0.6$ & $28.0 \pm 0.7$ & $32.3 \pm 0.8$ \\
\hline \multirow[t]{2}{*}{6} & 90 & $18.6 \pm 0.4$ & $21.6 \pm 0.4$ & $20.4 \pm 0.5$ & $23.1 \pm 0.5$ \\
\hline & 180 & $21.3 \pm 0.5$ & $23.2 \pm 0.5$ & $17.6 \pm 0.2$ & $19.8 \pm 0.4$ \\
\hline
\end{tabular}


Figure 8 shows the numerical simulation results of the temperature gradient $G_{z}$ (which is parallel to the [001] direction) in the solidification direction for the VR and TR samples at different withdrawal rates. The $G_{z}$ at a withdrawal rate of $6 \mathrm{~mm} \cdot \mathrm{min}^{-1}$ is greater than that at a withdrawal rate of $3 \mathrm{~mm} \cdot \mathrm{min}^{-1}$ [at the same solidification height of the same rod; Fig. 8(a) and (c)]. The $G_{z}$ is unstable and fluctuate, as observed previously the orientation evolution results (Figs. 2 and 4). The fluctuation range of $G_{z}$ at a withdrawal rate of $6 \mathrm{~mm} \cdot \mathrm{min}^{-1}$ is greater than that at $3 \mathrm{~mm} \cdot \mathrm{min}^{-1}$ [Figs. 8(b) and (d)]. Besides, by comparing with the simulation results shown in Fig. 7, it can be seen that the values of $G_{\mathrm{z}}$ are inversely related to the thickness of the mushy zone in the same casting. (a)

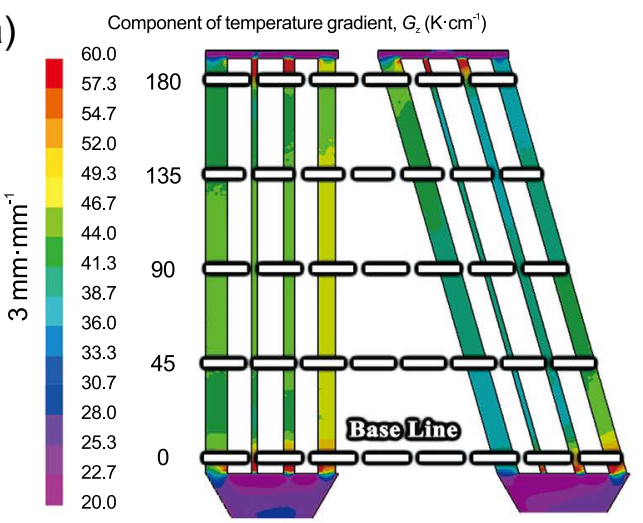

(c)

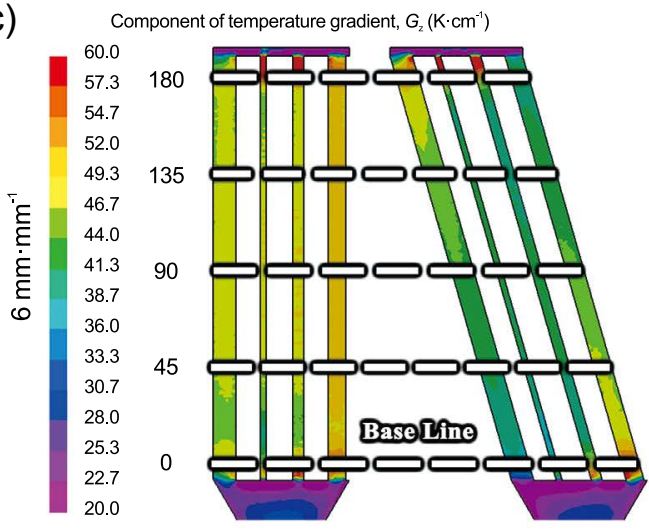

(b)

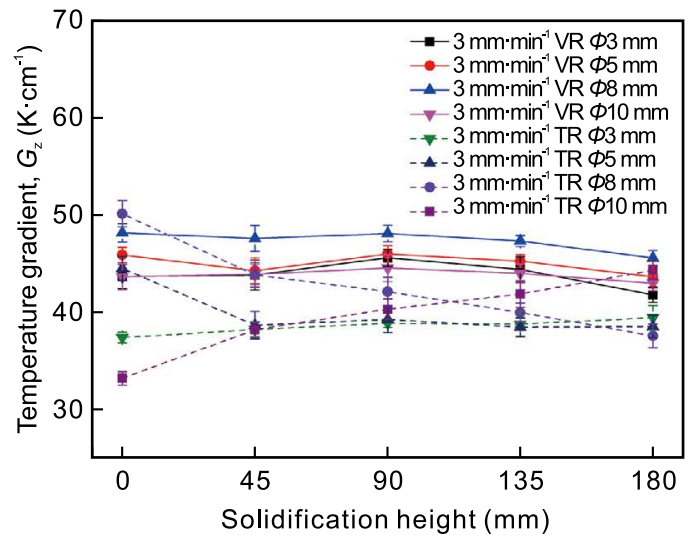

(d)

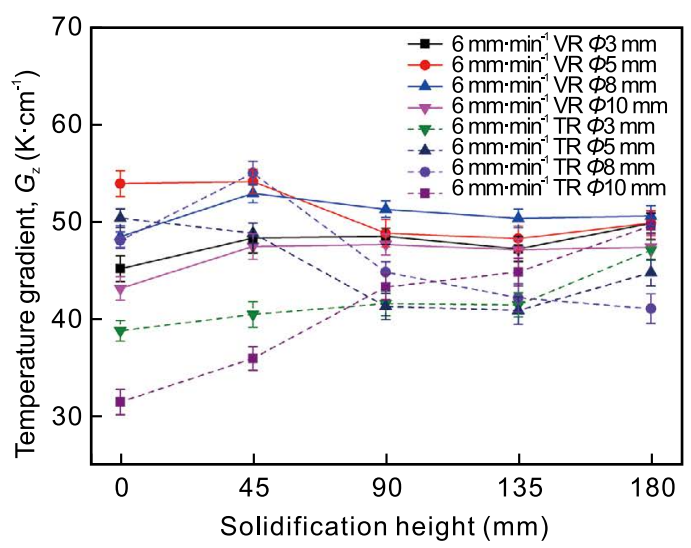

Fig. 8: Temperature gradient along [001] direction for VR and TR samples: (a) distribution of temperature gradient, $3 \mathrm{~mm} \cdot \mathrm{min}^{-1}$; (b) temperature gradient versus solidification height, $3 \mathrm{~mm} \cdot \mathrm{min}^{-1}$; (c) distribution of temperature gradient, $6 \mathrm{~mm} \cdot \mathrm{min}^{-1}$; (d) temperature gradient versus solidification height, $6 \mathrm{~mm} \cdot \mathrm{min}^{-1}$

\section{Discussion}

\subsection{Crystallographic orientation of primary dendrites}

From the orientation evolution results of a single-dendrite (Fig. 6), it is possible to infer the fluctuations of the primary dendrite orientations. According to studies ${ }^{[22-24]}$, during the process of dendrite growth, the value of the gradient angle $\alpha$ is not equal to zero. This is due to the incoherence between the direction of the heat flow and the preferred crystalline orientation; moreover, the actual growth directions of the dendrites lie between the temperature gradient direction and the preferred crystalline orientation. The preferred crystalline orientation of a material is constant and can be derived from its chemical composition and crystallographic structure. The growth direction of dendrites is mainly influenced by the thermal gradient.
Generally, the temperature gradient (relevant to the undercooling in solidification front) provides a driving force for the growth of dendrite during directional solidification. The direction of the temperature gradient is consistent with the heat flow direction and perpendicular to the tangential direction of the liquidus isotherms in the mushy zone ${ }^{[25-27]}$. In practice, the solid-liquid interface is a 3-dimensional space curved surface. However, due to its convenience for conducting observations and analyses, a 2-dimensional direction relationship diagram was employed (Fig. 9), where $G$ is the temperature gradient, $a$ is the preferred crystalline orientation, $\alpha$ is the included angle between $G$ and $a$, and $V_{\mathrm{g}}$ is the growth direction of the crystal. During the solidification process, the included angle $\alpha$ is very small. As for the face-centered cubic (FCC) structure of superalloys, the preferred crystalline orientation is [001] and the direction of $V_{\mathrm{g}}$ is close to the [001] direction. Actually, the $V_{\mathrm{g}}$ of the dendrites in the center of the rods is almost 


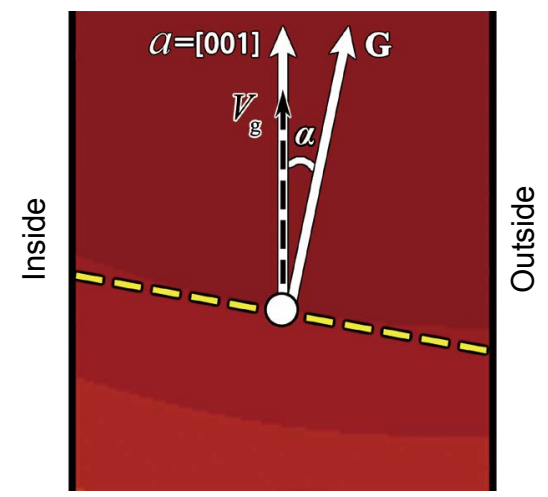

Fig. 9: Schematic illustration showing the relationship between morphology of liquidus isotherms in mushy zone and direction of temperature gradient

coincident with the preferred crystalline orientation $a$, while the $V_{\mathrm{g}}$ of the dendrites on both sides diverges and slightly deviates from the preferred crystalline orientation $a$. The reason for the slight deviation is the horizontal gradient from the center to the periphery. As for the TR samples, the melt above the solidification front on the diverging side (the left side in Fig. 7) is more than that on the converging side (the right side in Fig. 7); moreover, the horizontal cross-section size is larger and thicker than that of the VR samples, leading to tilted and concave isotherms. In addition, as for the thicker rods, the cooling rate is slower and the solidification time is longer than that of the thinner rods. Hence, the mushy zone thickness in the $\Phi 10 \mathrm{~mm}$ sample is larger than in the $\Phi 3 \mathrm{~mm}$ sample at the same solidification height.

The variation of $G$ is caused by some unstable factors that occur unavoidably during the directional solidification process, for example, the temperature field varying at any moment and the stress field variation caused by solidification shrinkage. Moreover, the geometry of the designed castings is also a nonnegligible influence factor that led to the variation of $G$. Such fluctuations of $G$ in the solidification direction make a positive contribution to the variation of shape and thickness of the mushy zone. In addition, the cooling rate in the solidification front varies with the temperature gradient $G$, which further impacts the growth and branching of dendrites. Due to the impact of interdendrite fluid impingement and shrinkage stress, the deviation of dendrite orientation might occur during the branching process. All factors mentioned above eventually result in the primary crystallographic orientation fluctuation of single-dendrites along the solidification direction.

The crystallographic orientation of the individual primary dendrites in the same cross-section was not completely identical (Fig. 3). This is owing to the efficiency of heat exchange on the outside surface towards the chill ring, which is subjected to intense cooling, being higher than that of the inside surface toward the central sprue ${ }^{[25,28]}$. Moreover, the neighboring components of the model assembly also exert a shading influence ${ }^{[29]}$. These factors resulted in an inhomogeneous distribution of the temperature gradient $G$ at the same solidification height [Figs. 8(a) and (c)]. This inconsistent temperature gradient induced the formation of curved isotherms [Figs. 7(c) and (d)]. At the curved solidliquid interface, the gradient angle $\alpha$ was different at different positions. The gradient angle $\alpha$ kept changing continuously from the shell wall to the central symmetry axis in the whole cross-section, leading to the different orientations of each primary dendrite.

\subsection{Evolution of crystallographic orientations}

The commonly referred to concept of single-crystal orientation equals to a statistical mean value, which is determined from all the individual dendrite orientations within the same crosssection. Figure 2 clearly shows that the evolution of these orientations is irregularly fluctuant in accord with the research of Newell ${ }^{[30]}$, perhaps due to the reasons listed below.

Firstly, the actual dendrites counted by EBSD within different sections are different and changeable, due to the propagation and elimination of dendrites caused by competitive growth during directional solidification. Based on the results shown in Fig. 5, the schematic diagram of dendrite count method is drawn in Fig. 10. The statistics of the dendrites counted in different cross-sections were not consistent. In the VR sample [Fig. 10(a)], the counted orientation of Dendrites No. 1 to No. 8 and No. 10 to No. 12 represents the singlecrystal orientation of Cross-section 1 . However, the number of dendrites in Cross-section 2 was different: the dendrites used for counting the orientation are No. 2, No. 3, No. 5 to No. 7 , No. 9 to No. 11 and No. 13. Since the dendrite orientations were not in the absolute [001] direction [Fig. 5(a)], the dendrites close to the ceramic shell wall (Dendrites No. 1 and No. 12) were overgrown by their adjacent internal dendrites (Dendrites No. 2 and No. 11) when meeting the shell wall ${ }^{[6]}$. Hence, the internal dendrites constantly overgrow the external dendrites by means of this elimination mechanism during the directional solidification process. Simultaneously, a portion of the protruding dendrites (Dendrites No. 5 and No. 7) and remelted dendrites (Dendrites No. 4 and No. 8) caused by the local thermosolutal convection form in the interdendritic regions, as reported in previous studies ${ }^{[30-33]}$.

(a)

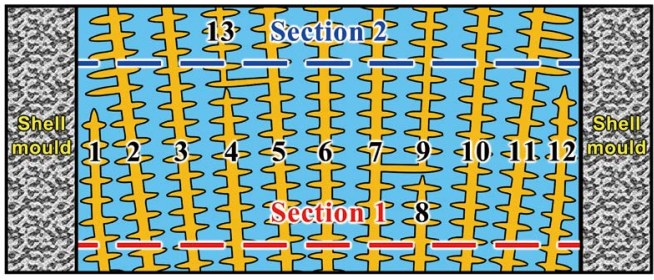

(b)

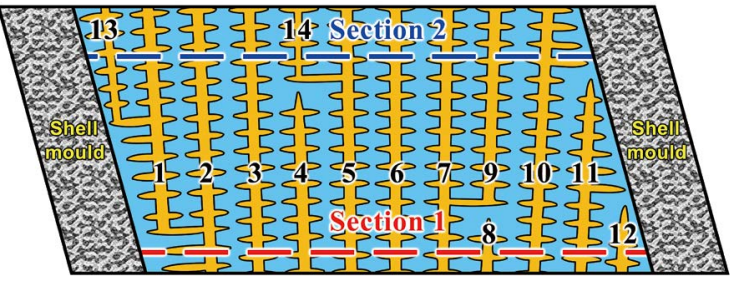

Fig. 10: Schematic illustration of dendrite statistical mode for VR (a) and TR (b) samples 
When the interdendritic solute-enriched liquid rises from both sides of a protruding dendrite, the vertical growth of the dendrite tip is inhibited and a knot forms at the dendrite tip. Then, the secondary dendrite arms branch laterally, blocking the growth of their neighboring primary dendrite arms. Eventually, the tertiary arms on the secondary dendrite arm grow vertically, becoming a new primary arm (Dendrites No. 13 and No. 9). In the TR sample [Fig. 10(b)], the branching mode of the dendrites is different with that in the vertical growth sample. The tilted solidification space gives rise to the formation of a gap zone between the dendrites and the shell wall; moreover, the secondary dendrite arms grow laterally to the high undercooling gap zone, and tertiary dendrites parallel to the original primary dendrite are developed (Dendrites No. 1 and No. 13). This process is analogous to the dendrite branching mode reported by Zhou ${ }^{[7]}$ and Wagner ${ }^{[34]}$. On the opposite side of the gap zone, the dendrites are efficiently eliminated by the intilted shell wall (Dendrites No. 11 and No. 12). The competitive growth mechanism of dendrites was similar in the TR sample (Dendrites No. 4, No. 5, No. 7, and No. 8). As a consequence, it can be concluded that the actual counted dendrites obtained when measuring the crystallographic orientation were not completely identical in cross-section at different solidification heights. Besides, because of the fluctuant evolution of the singledendrite primary orientation along the solidification direction, the orientation of the co-owned dendrites in Cross-sections 1 and 2 also changed. Based on the above analysis, the singlecrystal primary orientation at the height of Cross-section 1 was not strictly consistent with that at the height of Cross-section 2.

Secondly, the solidification space plays a significant role in the propagation and elimination of dendrites, further affecting the quantity of the actual counted dendrites. The ceramic shell wall provides little impact on the blocking of dendrites in the vertical solidification space [Figs. 5(a) and Fig. 6(a)], while the blocking effect of the tilted solidification space rods on the converging side is obvious [Figs. 5(b) and 6(c)]. On the diverging side, the secondary dendrites branched laterally into the undercooling melt located in the gap zone (between the ceramic shell wall and the dendrites); then, the ternary dendrites with the primary orientation are developed. New dendrites propagates continuously in this way. Therefore, the update frequency of the actual counted dendrites in the tilted solidification space is faster than in the vertical solidification space. This can possibly explain why the orientation fluctuation range of the TR samples was slightly larger than that of the VR samples.

The size of the gap zone is directly proportional to the deviation angle of the solidification space. When the gap zone is large enough, the vertical thermosolutal convection impinges on the long secondary dendrite arms (considered as cantilever beams) growing laterally. This process could lead to the formation of deviation, deformation and even fragmentation of dendrites. These abnormal dendrites keep growing, causing solidification defects (e.g., slivers, freckles, and stray grains) ${ }^{[35,36]}$. Besides, there is a potential risk of stray grain nucleation because of the high undercooling melt in the large gap zone. From the experimental results of this study, it can be inferred that, when the diameter of the GCs is smaller than $10 \mathrm{~mm}$, the $15^{\circ}$ deviation angle is still satisfied with the requirement of the single-crystal casting process; moreover, the fluctuation range of orientation is within the limits of acceptability. This is in line with our design anticipation.

Moreover, the effect of the withdrawal rate on the orientation cannot be ignored. According to the experimental results shown in Fig. 6, the orientation of single-dendrite fluctuates irregularly within a small range. Such orientation fluctuations could have been caused by variations in the cooling rate, as well as in the shape and thickness of the mushy zone (arising from the withdrawal rate). According to a previous work ${ }^{[37]}$, the cooling rate varies with the decrease of the heat transfer coefficient in the vertical direction; moreover, the shape and thickness of the mushy zone are influenced by the location against the radiation baffle and the temperature gradient, respectively ${ }^{[26,38]}$, as reflected by the following equation:

$$
L=\Delta T / G=\left(T_{\mathrm{L}}-T_{\mathrm{S}}\right) / G
$$

where $L$ is the thickness of the mushy zone, mm, $\Delta T$ is the temperature difference between the solidus $T_{\mathrm{S}}$ and the liquidus $T_{\mathrm{L}}$, and $G$ is the temperature gradient in the mushy zone. From Eq. (1), the temperature gradient $G$ increases as the thickness of the mushy zone $L$ decreases. This is in good agreement with the results shown in Fig. 8.

With the increase of the withdrawal rate, $L$ becomes smaller and the isotherms become more concave and tilted (Fig. 7). These curved and tilted isotherms in the mushy zone reflect a larger difference in the temperature gradient $G$ among different positions, and a further enlargement of the difference in the actual growth direction of all dendrites. Therefore, competitive growth occurred between dendrites with favorable and unfavorable crystallographic orientations ${ }^{[39-41]}$. Hence, a high withdrawal rate intensifies the degree of dendritic competitive growth in the same sample, increasing the update frequency of the actual counted dendrites. This, maybe, is the reason that the fluctuation range of orientation in the samples at withdrawal rate of $6 \mathrm{~mm} \cdot \mathrm{min}^{-1}$ is slightly larger than that at $3 \mathrm{~mm} \cdot \mathrm{min}^{-1}$.

In addition, the cross-section size also has an effect on orientation fluctuation. The shape of the solid-liquid interface in different cross-section size castings is discrepant under the same withdrawal rate, leading to differences in the temperature gradient $G$. For small cross-section size castings, there is a smaller difference in transverse heat extraction between the inside and outside of the mold shell, resulting in a relatively flat solid-liquid interface and a consistent $G^{[26]}$. However, for large cross-section size castings, a larger inside/outside difference in transverse heat extraction is observed. Meanwhile, as the amount of metal melt increases in the mushy zone, the heat in the center could not be extracted rapidly, causing the temperature in the center to be higher than that on both sides of the rod. A large cross-section size aggravates the temperature difference at the mushy zone among the inside, the middle and 
the outside of the rod. Based on the points mentioned above, a curved and tilted solid-liquid interface and an inconsistent temperature gradient $G$ are obtained for the large crosssection size rod. These elements lead to great difference in the crystallographic orientations of the actual counted dendrites, thereby intensifying the competitive growth of dendrites and speeding up the update frequency of dendrites. Thus, the orientation fluctuation in the large cross-section is slightly greater than that in the small sized cross-section. However, the differences in orientation fluctuation between different sized cross-sections are not apparent in the experiments in this study, because the diameters of tested samples do not exceed $10 \mathrm{~mm}$.

Finally, due to the use of the spiral grain selection method, the initial crystallographic orientation of the secondary dendrites is random. From the evolution results of the crystallographic orientation (Figs. 2 and 4), the secondary dendrite orientations are fluctuate, and the orientation fluctuation range of the secondary dendrites is at the same level as that of the primary dendrites. This is owing to the crystallographic characteristics of dendrites: the secondary dendrites always grow perpendicularly to the primary dendrites. Therefore, the secondary dendrites deviate when the primary dendrites deviate.

In general, the suitable $\mathrm{LAB}$ range from $2^{\circ}$ to $15^{\circ}$ in single crystal castings can be accepted by manufacturing standards ${ }^{[42]}$. In regard to single-crystal superalloys (e.g., Rene N, CM186LC, and PWA1483), the tolerance angle of the LABs in superalloy castings is larger than $6^{\circ}{ }^{[43]}$. However, the maximum fluctuation range of the crystallographic orientation is less than $4^{\circ}$ in this work. Therefore, the rods designed in this study can be used as GCs for the manufacturing of single crystal turbine blades.

\section{Conclusions}

The crystallographic orientation of single-crystal GCs with different cross-section sizes and deviation angles were determined at different solidification heights. The characteristics of dendrites and crystallographic orientation evolution along the solidification direction were analyzed and discussed. Based on the results obtained, the following conclusions are drawn:

(1) The crystallographic orientation of single crystals corresponds to the statistical average value of all the individual dendrite orientations in the selected cross-section. Both the primary and secondary orientations of single-crystal GCs fluctuate irregularly within a small range (by less than $4^{\circ}$ ).

(2) The fluctuation range of the crystallographic orientation along the solidification direction becomes larger with the increase of the withdrawal rate.

(3) The cross-section size (maximum $10 \mathrm{~mm}$ ) of singlecrystal GCs has an insignificant influence on the evolution of the crystallographic orientation along the solidification direction.

(4) Rods with diameters of 3-10 mm and deviation angles of $0-15^{\circ}$ from the solidification direction can be sufficient for GCs by adopting proper solidification process parameters.

\section{Acknowledgements}

This work was supported by the National Natural Science Foundation of China (No. 51674235) and the National Key Research and Development Program of China (Nos. 2017-VI0001-0070 and 2017-VI-0019-0091).

\section{References}

[1] Kim I S, Choi G G, Seo S M, et al. Influence of heat treatment on microstructure and tensile properties of conventionally cast and directionally solidified superalloy CM247LC. Materials Letters, 2008, 62(6-7): 1110-1113.

[2] Yang W C, Hu S S, Huo M, et al. Orientation controlling of Ni-based single-crystal superalloy by a novel method: Grain selection assisted by un-melted reused seed. Journal of Materials Research and Technology, 2019, 8(1): 1347-1352.

[3] Wang X D, Yang Z, Gao Q, et al. Effect of long-term thermal exposure on microstructure and creep properties of DD5 single crystal superalloy. China Foundry, 2021, 18(3): 185-191.

[4] Huang M, Zhang G, Wang D, et al. Dendritic branching patterns in platforms of complex $\mathrm{Ni}$-based single crystal castings. China Foundry, 2019, 16(2): 110-117.

[5] Han D Y, Jiang W G, Xiao J H, et al. Investigation on freckle formation and evolution of single-crystal nickel-based superalloy specimens with different thicknesses and abrupt cross-section changes. Journal of Alloys and Compounds, 2019, 805: 218-228.

[6] Aveson J W, Tennant P A, Foss B J, et al. On the origin of sliver defects in single crystal investment castings. Acta Materialia, 2013, 61(14): 5162-5171.

[7] Zhou Y Z. Formation of stray grains during directional solidification of a nickel-based superalloy. Scripta Materialia, 2011, 65(4): 281-284.

[8] Ma D X, Wang F, Wu Q, et al. Temperature evolution and grain defect formation during single crystal solidification of a blade cluster. China Foundry, 2017, 14(5): 456-460.

[9] Li Y F, Liu L, Huang T W, et al. Simulation of stray grain formation in Ni-base single crystal turbine blades fabricated by HRS and LMC techniques. China Foundry, 2017, 14(2): 75-79.

[10] Ma D X. Novel casting processes for single-crystal turbine blades of superalloys. Frontiers of Mechanical Engineering, 2017, 13(1): 3-16.

[11] Yoshinari A, lijima $\mathrm{K}$, Kodama $\mathrm{H}$, et al. Nickel base superalloys single crystal growth technology for large size buckets in heavy duty gas turbines. In: Proc. International Gas Turbine and Aeroengine Congress and Exposition, Orlando, USA, 1991: $1-6$.

[12] Toloraya V N, Demonis I M, and Ostroukhova G A. Formation of single-crystal structure of large-size cast GET and GUT turbine blade in facilities for high gradient directed crystallization. Metal Science and Heat Treatment, 2011, 53(1-2): 24-31.

[13] Vehn M M T, Dedecke D, Paul U, et al. Undercooling related casting defects in single crystal turbine blades. Superalloys 1996: 471-496.

[14] Newell M, D'Souza N, and Green N R. Formation of low angle boundaries in Ni-based superalloys. International Journal of Cast Metals Research, 2013, 22(1-4): 66-69.

[15] Yue Z F, and Lu Z Z. The influence of crystallographic orientation and strain rate on the high-temperature low-cyclic fatigue property of a nickel-base single-crystal superalloy. Metallurgical and Materials Transactions A, 1998, 29A: 10931099 
[16] Kakehi K, Latiefn F H, and Sato T. Influence of primary and secondary orientations on creep rupture behavior of aluminized single crystal Ni-based superalloy. Materials Science and Engineering A, 2014, 604: 148-155.

[17] Suzuki S, Sakaguchi M, and Inoue H. Temperature dependent fatigue crack propagation in a single crystal Ni-base superalloy affected by primary and secondary orientations. Materials Science and Engineering A, 2018, 724: 559-565.

[18] Newell M, Devendra K, Jennings P A, et al. Role of dendrite branching and growth kinetics in the formation of low angle boundaries in Ni-base superalloys. Materials Science and Engineering A, 2005, 412(1-2): 307-315.

[19] Hu S S, Liu L, Yang W C, et al. Formation of accumulated misorientation during directional solidification of $\mathrm{Ni}$-based single-crystal superalloys. Metallurgical and Materials Transactions A, 2019, 50(4): 1607-1610.

[20] Glicksman M E. Principles of solidification. New York: Springer, 2011: 305-343.

[21] Rzyankina E, Szeliga D, Mahomed N, et al. Investigation of the effect of solidification velocity on the quality of single crystal turbine blades. Applied Mechanics and Materials, 2013, 372: 54-61.

[22] Li J J, Wang Z J, Wang Y Q, et al. Phase-field study of competitive dendritic growth of converging grains during directional solidification. Acta Materialia, 2012, 60(4): 1478-1493.

[23] Pocheau A, Deschamps J, and Georgelin M. Dendrite growth directions and morphology in the directional solidification of anisotropic materials. JOM, 2007, 59: 71-76.

[24] Deschamps J, Georgelin M, and Pocheau A. Growth directions of microstructures in directional solidification of crystalline materials. Physical Review E, 2008, 78: 011605.

[25] Szeliga D, Kubiak K, Motyka M, et al. Directional solidification of Ni-based superalloy castings: Thermal analysis. Vacuum, 2016, 131: 327-342.

[26] Kurz W, Fisher D Z. Fundamentals of solidification. Aedermannsdorf, Switzerland: Trans. Tech. Publications, 1986: 47-96.

[27] Dantzig J A, Rappaz M. Solidification. Lausanne, Switzerland: EPFL Press, 2009: 287-342.

[28] Franke M M, Hilbinger M, Heckl A, et al. Effect of thermophysical properties and processing conditions on primary dendrite arm spacing of nickel-base superalloys-numerical approach. Advanced Materials Research, 2011, 278: 156-161.

[29] Ma D X, Wang F, Wu Q, et al. Innovations in casting techniques for single crystal turbine blades of superalloys. In: Proceedings of the 13th International Symposium on Superalloys, The Minerals, Metals \& Materials Society (TMS), 1996: 237-246.

[30] Shevchenko N, Roshchupkina O, Sokolova O, et al. The effect of natural and forced melt convection on dendritic solidification in Ga-In alloys. Journal of Crystal Growth, 2015, 417: 1-8.
[31] Yang C, Xu Q Y, and Liu B C. Study of dendrite growth with natural convection in superalloy directional solidification via a multiphase-field-lattice Boltzmann model. Computational Materials Science, 2019, 158: 130-139.

[32] Miller J D, and Pollock T M. Stability of dendrite growth during directional solidification in the presence of a non-axial thermal field. Acta Materialia, 2014, 78: 23-36.

[33] Brundidge C L, Miller J D, and Pollock T M. Development of dendritic structure in the liquid-metal-cooled, directionalsolidification process. Metallurgical and Materials Transactions A, 2011, 42(9): 2723-2732.

[34] Wagner A, Shollock B A, and McLean M. Grain structure development in directional solidification of nickel-base superalloys. Materials Science and Engineering A, 2004, 374(1-2): 270-279.

[35] Huang $Y$ Q, Shen J, Wang D, et al. Formation of sliver defect in Ni-based single crystal superalloy. Metallurgical and Materials Transactions A, 2020, 51(1): 99-103.

[36] Huang $Y Q$, Shen J, Wang D, et al. Evolution of sliver defect in Ni-based single crystal superalloy. Metallurgical and Materials Transactions A, 2020, 51(12): 6364-6372.

[37] Elliott $A \mathrm{~J}$ and Pollock $\mathrm{T} \mathrm{M}$. Thermal analysis of the bridgman and liquid-metal-cooled directional solidification investment casting processes. Metallurgical and Materials Transactions A, 2007, 38(4): 871-882.

[38] Miller J D and Pollock T M. The effect of processing conditions on heat transfer during directional solidification via the Bridgman and liquid metal cooling processes. Metallurgical and Materials Transactions A, 2013, 45(1): 411-425.

[39] Zhou Y Z, Volek A, and Green N.R. Mechanism of competitive grain growth in directional solidification of a nickel-base superalloy. Acta Materialia, 2008, 56(11): 2631-2637.

[40] Liu Y, Liu M, Yu D E, et al. Dependence of competitive grain growth on secondary dendrite orientation during directional solidification of a Ni-based superalloy. Metallurgical and Materials Transactions A, 2013, 44(11): 5113-5121.

[41] Meng X B, Lu Q, Zhang X L, et al. Mechanism of competitive growth during directional solidification of a nickel-base superalloy in a three-dimensional reference frame. Acta Materialia, 2012, 60(9): 3965-3975.

[42] Huo M, Liu L, Yang W C, et al. Formation of low-angle grain boundaries under different solidification conditions in the rejoined platforms of $\mathrm{Ni}$-based single crystal superalloys. Journal of Materials Research, 2019, 34(2): 251-260.

[43] Wang Q J, Song J X, Wang D G, et al. Quantified relation between grain boundary angle and interfacial stability of PWA1484 superalloy during thermal exposure. Metallurgical and Materials Transactions A, 2020, 51: 380-389. 